\title{
Ensuring safe access to medication for palliative care while preventing prescription drug abuse: innovations for American inner cities, rural areas, and communities overwhelmed by addiction
}

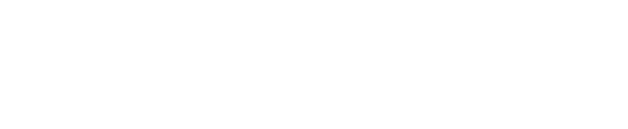

\section{Richard B Francoeur \\ School of Social Work, Adelphi University, Garden City, NY, USA; Center for the Psychosocial Study of Health and Illness, Columbia University, New York, NY, USA}

\begin{abstract}
This article proposes and develops novel components of community-oriented programs for creating and affording access to safe medication dispensing centers in existing retail pharmacies and in permanent or travelling pharmacy clinics that are guarded by assigned or off-duty police officers. Pharmacists at these centers would work with police, medical providers, social workers, hospital administrators, and other professionals in: planning and overseeing the safe storage of controlled substance medications in off-site community safe-deposit boxes; strengthening communication and cooperation with the prescribing medical provider; assisting the prescribing medical provider in patient monitoring (checking the state prescription registry, providing pill counts and urine samples); expanding access to lower-cost, and in some cases, abuse-resistant formulations of controlled substance medications; improving transportation access for underserved patients and caregivers to obtain prescriptions; and integrating community agencies and social networks as resources for patient support and monitoring. Novel components of two related community-oriented programs, which may be hosted outside of safe medication dispensing centers, are also suggested and described: (1) developing medication purchasing cooperatives (ie, to help patients, families, and health institutions afford the costs of medications, including tamper- or abuse-resistant/deterrent drug formulations); and (2) expanding the role of inner-city methadone maintenance treatment programs in palliative care (ie, to provide additional patient monitoring from a second treatment team focusing on narcotics addiction, and potentially, to serve as an untapped source of opioid medication for pain that is less subject to abuse, misuse, or diversion).
\end{abstract}

Keywords: controlled substances, diversion, drug abuse, inner-city, methadone, methadone maintenance treatment, pain, palliative care, pharmacy, prescription drugs, rural, safety, tamper- and abuse-resistant drugs, TARD, transportation

Converging economic factors and bioethical concerns will increasingly direct the options for pain control that will be made available to patients, families, and health care institutions, especially in inner-city and rural settings. Health insurers face pressures to limit coverage of less cost-effective options for pain-relieving opioids and other medications, at the same time that health institutions, patients, and families are restricting the level of out-of-pocket, or nonreimbursed, costs that they will bear. ${ }^{1}$ However, many other costs, difficult to assess in economic terms, must also be considered, especially the repercussions from drug abuse and diversion on patients, families, physicians, communities, and society. Alarmingly, prescription
Correspondence: Richard B Francoeur School of Social Work,

Adelphi University, I South Avenue,

Garden City, NY II530, USA

Tel + I 917254727 I

Email francoeur@adelphi.edu 
drug abuse is now the second leading cause of unintended death in the United States. ${ }^{2}$ Determining which options for pain management are humane, affordable, and acceptable in any given context requires assessment of: (1) addiction and drug diversion not only by patients but by their caregivers, family, and social contacts; (2) personal safety of the patient, caregiver, and family members from drug dealers; and (3) perceptions of stigma by the patient, caregiver, and family.

In inner-city and rural areas, health providers often find it difficult to obtain medications, face higher costs for medications, and struggle to ensure their safe delivery. Restrictions by physicians in prescribing medication, ${ }^{3,4}$ and by pharmacists in stocking them, ${ }^{5,6}$ exist where there are serious concerns over illegal drug trafficking and drugrelated crime. The United States Drug Enforcement Agency (DEA) prosecutes physicians when patients divert drugs that were prescribed to relieve pain, ${ }^{7}$ which reinforces physician reluctance to prescribe pain medications in sufficient quantities when there may be any possibility for diversion or abuse. ${ }^{3,4}$ On the other hand, it may be anticipated that patients and caregivers could increasingly come to fear for their safety and therefore avoid well stocked pharmacies in communities, regardless of their geography, that have experienced increased incidents of hold-ups, shootings, or other violent crime. ${ }^{8}$

\section{Retail pharmacies and pharmacy clinics in the community: innovations for safety and prevention}

In response to evidence that inner-city pharmacies do not stock opioids due to the potential for assault, theft, and vandalism $^{5,6}$ - and as options for any geographic location in which crime or shootings at retail pharmacies may drive away customers ${ }^{8}$ - this article presents a vision of alternative pharmacy options, that involve improving the safety of retail pharmacies and pharmacy clinics in the community. These alternative options merit serious consideration for research and program development.

Community pharmacists in inner-city and rural areas may be more willing to dispense opioid medications and other controlled substances as long as (1) an on-duty police officer can be assigned or an off-duty police officer hired to provide on-site protection; and (2) supplies of opioid medications can be safely stored offsite, with the police officer trained to obtain and transport, on a periodic basis, the types and amounts that the pharmacist anticipates will be needed. In this option, community pharmacists would dispense these medications at a retail pharmacy, or at a permanent or traveling pharmacy clinic held at other community locations, such as church basements, fraternal organizations (eg, Veterans of Foreign Wars or Masonry halls), town halls, hospitals and outpatient hospital clinics, nursing homes and assisted living sites, and farm supply stores. The presence of a police officer in a retail pharmacy, or at common community locations that host a pharmacy clinic, may avoid much of the stigma that patients and caregivers may be likely to experience compared, for instance, to locating a retail or clinic pharmacy at a local police, sheriff, or state police station, although research evidence is needed to confirm this reaction. For this innovative type of program to work, several features need to be adopted.

First, safety concerns may mean that opioid medications and other controlled substances should be delivered to the local police or sheriff station, where they would be handled, labeled, and stored by the pharmacist. (These medications may include lower-priced, mail-order medications from Internet pharmacies purchased as part of a community health consortium, as discussed below.) The pharmacist and police officer, or the police officer alone, would then transport medications and store them in safe deposit boxes obtained for this purpose that are located in restricted access areas at police/sheriff stations, banks, and/or post offices. (Safe deposit boxes are located not only at banks, but at post offices in some communities, ${ }^{9}$ which would appear to be an option for consideration in others, along with locating safe deposit boxes in police stations). These locations should be dispersed across different geographic regions of the inner-city, rural area, or other type of community. The pharmacist must also train the police officer in the proper procedures for identifying and handling these medications and transporting them from the safe deposit boxes to the retail pharmacy or pharmacy clinic.

Second, these safe deposit boxes should only be accessible by the pharmacist and the police officer. The pharmacist, perhaps accompanied by the police officer, would stock safe deposit boxes with medication. (Subsequently, the police officer would travel alone to select periodically only the needed types and quantities of medication from these safe deposit boxes for delivery to the community pharmacy or pharmacy clinic.) A search of the literature and the Internet by the author did not reveal state or federal laws that prohibit legally obtained controlled substances from being stored in safe deposit boxes. Moreover, legal drugs are typically not among the restrictions listed on agreement forms for use of safe deposit boxes at banks. ${ }^{10,11}$ (Banks are also free to tailor their agreement 
forms to these special circumstances in order to better meet the needs of the bank and the institutions represented by the pharmacist and police officer.) In addition, new biometric instant recognition technologies, such as three-dimensional reading of a hand's size and shape, provide additional safety for preventing robberies, ${ }^{12}$ although traditional safe deposit boxes (ie, accessed using a key after an attendant unlocks the room with a separate key) may be considered sufficient, especially in smaller communities.

Third, the police officer should arrive with the appropriate medications, and in the required quantities, sufficiently in advance of when the pharmacist begins prescribes medications during scheduled appointments with patients or their caregivers. (Limited quantities of these medications should be dispensed during each visit by a patient or their caregiver in order to reduce the chances that they will become targets for assault and theft of their medication, and to reduce the chances that they will divert or abuse the medication. As discussed later in this article, pill counts of remaining medication could be required before renewing a prescription, and increases in prescription quantities over time could be approved when pill counts do not suggest problems.) Scheduling appointments during restricted hours and days that are set aside for prescribing opioids and other controlled substances should improve cost feasibility since the police officer will only provide on-site protection during limited hours.

Additional security features already used by some pharmacies include a locked entrance with an outdoor buzzer that can be viewed by camera, which permits access only to those with a scheduled appointment, and a sign on the outside door stating that the pharmacy does not stock opioid pain medications, such as oxycodone, nor other controlled substances. Local ordinances may prohibit loitering near the pharmacy, especially when controlled substances are prescribed, and in some cases, police officers may obtain restraining orders to prevent certain individuals with drug addiction or diversion involvement from approaching the pharmacy.

These program components may be adopted not only by retail pharmacies but by pharmacy clinics (including temporary or rotating clinics) that would likewise improve safety of medication dispensing by using assigned (ie, on-duty) or hired (ie, off-duty) police officers. Pharmacy clinics operated by the local hospital provide an important alternative to the retail pharmacy, which may not exist or be feasible to operate, especially in rural areas. Additional safety protection may result to the extent that community locations for these clinics are not selected according to a publicized and predictable schedule and to the extent that patients and caregivers with scheduled appointments can keep the locations confidential. In rural areas with scattered populations and with vast distances that need to be served, the costs of using police officers for a pharmacy clinic (which would need to cover their traveling times) are likely to be prohibitive. A travelling clinic may be more feasible in inner-city areas with good transportation options and in semirural regions punctuated by areas that are more densely populated.

Funding for prevention from the recent federal health care reform legislation, the Affordable Care Act, as well as Medicare, might be tapped since close monitoring for medication adherence could provide more cost-effective management of disease conditions and prevent pain and symptoms from becoming out of control due to medication mismanagement or drug diversion. The DEA and local philanthropies may be additional sources of potential funding to subsidize the costs of assigning a police officer, or hiring one off-duty, who would obtain and transport controlled substance medications from community safe-deposit boxes to either a retail pharmacy or a pharmacy clinic, as well as provide on-site police protection. These police officer functions constitute two critical program components for safe dispensing of medication and prevention of prescription drug abuse.

Cost savings from online purchasing of wholesale medications in bulk as a mail-order from a consortium of health care institutions could also provide funding and operational advantages. The consortium could involve pharmacies located in the community and within hospitals, and that serve nursing homes, visiting nurse services, and hospices. Some cash-strapped hospitals and visiting nurse associations in rural areas have organized into consortiums that order medications online from large, wholesale medicine-ordering cooperatives that bypass the middle retail pharmacy. ${ }^{13}$ However, a consortium might include urban, suburban, and rural institutions within the same geographic regions. ${ }^{14}$ Wholesale, online medicine-ordering cooperatives can ship medications directly to home addresses, hospitals and neighborhood pharmacies, ${ }^{13}$ and other locations. Social workers, health providers, and health administrators from rural and inner-city hospitals, visiting nurse associations, and palliative care programs could advocate for this option to reduce unreimbursed medication expenditures borne by patients, families, and the health institution. ${ }^{14}$

\section{Transportation issues: innovations in service delivery and reimbursement}

Some patients and designated caregivers from sprawling inner cities and vast rural counties are likely to find it 
prohibitive to travel alone, contend with complicated public transportation systems, or drive long distances to retail pharmacies or pharmacy clinics. In some situations, a bus, van, or car option could transport residents to the retail pharmacy or travelling pharmacy clinic, as well as the return trip home. The additional costs to the community of this transportation option may be offset to some extent by a transportation charge on a sliding scale based on patient income, or by a fare in the case of the use of preexisting public transportation. A transportation option may be more feasible financially to operate in inner-city areas, however in semirural regions, it may still be worthwhile to assess whether cost savings in averted or delayed hospital admissions could justify a car or van option. Moreover, these additional transportation costs could be argued in many cases to offset the eventual inpatient hospital costs that are borne by the community, including uncompensated care and the share of Medicaid reimbursement financed through local and state taxation. Late referrals of individuals with exacerbated illness, who present with long-neglected pain and other symptoms become more difficult and more expensive to control, in contrast to when residents obtain early and sustained palliation and control of their symptoms. ${ }^{15,16}$ Advocacy with public and private insurers to cover these transportation expenses as part of preventive/palliative care should be considered since these health insurers ultimately stand to save money - not necessarily over patient life spans (since preventive care and symptom palliation might cause a patient to live longer) - but over the more critical short-term period of the annual fiscal year. To health insurers, the annual fiscal year is the bottom-line period for assessing net profitability and total revenues from annual enrolments in private plans as well as annual public reimbursements financed through local, state, and federal taxation. Social workers, other clinicians, and staff from hospital reimbursement departments could work together to advocate for transportation service delivery and reimbursement.

Options for round-trip transportation merit consideration that would offer the only means of access by individuals from underserved groups in the community. The lowest cost transportation option may be based on a predetermined limited schedule of operation, a direct route, and express service with only a single stop between the retail pharmacy or travelling clinic and a specific location in the community. This lowest cost approach may make it feasible to take advantage of untapped opportunities to reach concentrated populations of underserved populations.

For instance, homeless individuals, including those who reside at shelters, cannot safely store a prescription supply of opioids and other medications and a frequent need to migrate may compromise safety in other ways. Safety issues are especially salient when a homeless person with a supply of opioid medications stays at a shelter, since they may become magnets for assault and violence from other residents at these facilities who are actively abusing drugs or involved in diversion activities. These difficulties may mean that at least some homeless individuals, and their physicians, would welcome the option to obtain and consume medications within the safety of a retail or hospital pharmacy or a traveling pharmacy clinic guarded by a police officer, as long as they felt reassured that the officer would not harass them. Community groups could be mobilized to help set up round-trip transportation between a local shelter and the pharmacy or pharmacy clinic in order to prevent loitering and provide an option for temporary lodging after medications are consumed. In addition, when a hospital pharmacy has continuous hours of operation, a locked box of medications could be kept in the dispensing room for a homeless individual to travel there in order to consume prescribed medications onsite and/or to obtain medications for a short period. It is unclear, however, whether tamper- or abuse-resistant or deterrent (TARD) medications, discussed next, would afford greater safety to homeless individuals so that they could obtain a larger supply of medications for a longer period.

\section{Drug formulation improvements}

Nursing homes are other settings in inner-city and rural communities that are vulnerable to medication diversion and abuse. Because nursing homes operate without doctors or pharmacies on their premises, pain medications are delayed due to the mandate by the DEA that pain medications be prescribed only after written signatures from physicians are faxed to off-site pharmacies. ${ }^{17,18}$ Pain medication delays are especially lengthy for residents with pain exacerbation in the middle of the night or who are in transition from the hospital. These mandates are designed to lower the potential for drug theft and abuse by nursing home staff. New TARD formulations of narcotics are designed to discourage prescription drug abuse and diversion. ${ }^{1}$ Advocacy efforts to gain exceptions to this DEA mandate could be more compelling when 
they are sought for situations in which TARD formulations are used.

However, TARD formulations tend to be more expensive, posing greater financial burden for underinsured and lower socioeconomic populations. ${ }^{1,19}$ It has been suggested elsewhere that hospitals, hospices, and nursing homes from inner-city, urban, suburban, and rural areas could engage collectively as a consortium to allow online mail-order purchases of a wider range of medications, including TARD formulations, at reduced costs to these health facilities and to patients and families. ${ }^{14}$ Cost savings from medications purchased as a consortium could be used to (1) offset financial burden by patients, families, and health facilities in meeting unreimbursed medication expenses; and/or (2) help finance service delivery innovations such as a round-trip transportation service or a permanent or travelling safe medication dispensing center within the community. Social workers and health providers within the same geographic region could network with each other to assess the potential to work towards this kind of macrosystem change for their health institutions and communities. $^{14}$

TARD preparations of opioid medications are specially formulated to be difficult to melt or crush so that they cannot be injected or inhaled. ${ }^{1,19}$ Other formulations become inactivated when melted or crushed, which releases a second drug with significantly greater affinity for the microopioid receptor, thus displacing the opioid from binding to the receptor. A methadone/naloxone 50:1 preparation, for instance, was developed in Australia to eliminate diversion and injection of prescribed methadone. ${ }^{20}$ An inexpensive and synthetic opioid, methadone is likely to be more cost-effective when formulated as a TARD preparation than TARD preparations based on other opioids, which could result in significant savings for health institutions, patients, and families. Other TARD options, such as sublingual buprenorphine-naloxone tablets or subdermal buphrenorphine implants, ${ }^{21}$ are based on opioids with a better safety profile than methadone, which is contraindicated in certain patients due to the potential for life-threatening interactions with other medications, treatments, or conditions.

Despite the enhanced safety and affordability that some TARD medications could provide, there is no evidence that TARD formulations will prevent patients who abuse or divert opioid drugs from seeking to manipulate medical personnel into prescribing excess amounts, or stronger than necessary, drugs. ${ }^{19}$ Still, TARD formulations may make it more difficult for patients, family members, or friends to abuse them and less worthwhile to divert them. These advantages may hold promise for curtailing thefts of medications by nursing home employees and from community pharmacies, which could be easily monitored to determine whether medication thefts decrease when TARD formulations are used.

\section{Strategically linking palliative pharmacotherapy to community programs and resources Methadone maintenance treatment programs}

The fact that opioid medications for pain are narcotics may lead patients and family members to feel stigma, especially in inner-city and rural areas that struggle with drug addiction and are caught up with efforts to curtail black market drug diversion activities. Patients receiving methadone, in particular, to relieve pain have disclosed perceptions of stigma. ${ }^{22,23}$ This stigma relates to the parallel use of methadone at methadone maintenance treatment programs (MMTPs) to manage craving in those with the disease of addiction; the mention of methadone as a medication for pain is often interpreted as a message that we are treating an unacknowledged but presumed addiction in the patient. Social workers and health providers who are aware of those faulty attributions can assist patients and families to consider use of methadone for pain by raising concerns, acknowledging fears, exploring any addiction history, and educating about the use of methadone for pain management. Without intervention, it is unclear whether stigma will remain sufficiently strong to override the use of methadone for chronic pain, especially when patients are underinsured for more costly opioid prescriptions or neighborhood pharmacies do not stock them. ${ }^{6}$

Ironically, the very source of this stigma may provide another option to TARD preparations for safe and affordable methadone to treat pain. In some inner-city areas, MMTPs already serve individuals who are addicted to euphoriainducing narcotics. These clinics merit serious consideration as an untapped source of opioid medication for the palliative relief of pain that is potentially less subject to diversion than other delivery systems for opioid medications. Patients with HIV (human immunodeficiency virus)/AIDS (acquired immune deficiency syndrome) and already receiving treatment for narcotic addiction at MMTPs have been treated successfully with additional methadone in these programs to relieve diverse chronic pain syndromes. ${ }^{24}$ Moreover, it should also be considered whether methadone delivered at 
an MMTP could provide a safer and more attractive option not only for patients in pain with narcotics addictions, but for other patients (1) whose physicians would otherwise resist prescribing opioids in sufficiently high doses needed to relieve pain; and (2) who may not have safe access, or financial access, to opioids for use at home, including homeless populations. ${ }^{25}$

However, state government regulations often restrict MMTPs to treating narcotics addiction (and not pain relief). ${ }^{25}$ Barriers to access include daytime hours of operation, and automatic daily treatment regimens of 5-7 days of the week, which employed patients and family caregivers cannot meet, as well as payment mechanisms set up only for Medicaid reimbursement (ie, other health insurance or out-of-pocket payment cannot be accommodated). Even so, the changing makeup of the population with narcotics addictions could provide new opportunities for these clinics; for instance, there appears to be bipartisan interest in the chronic physical and mental health problems of veterans returning from Afghanistan and Iraq. Affording veterans with improved access to these clinics may reduce stigma and enhance the community profiles of these programs, which could build momentum to serve more clients with narcotics addictions.

The involvement of two treatment teams in patient monitoring and professional consultation is advantageous and recommended. ${ }^{4}$ Even when MMTPs do not deliver additional methadone to relieve pain, patients attending these clinics to treat narcotics addiction require close coordination by the palliative care team (which may be using opioid medication to treat pain) and the MMTP treatment team. Drug screenings such as urine drug screens, recommended as part of the Utah clinical guidelines on prescribing opioids for treating pain, ${ }^{26}$ could be performed by each team. Although clinical practice recommendations provide maximum doses to reduce the risk of overdose and death, ${ }^{22,27-29}$ physicians may not be well versed about how methadone can interact with medications and present risks for overdose and death. Thus, only experienced palliative care physicians committed to close patient monitoring should prescribe methadone to relieve pain. ${ }^{26}$

The assistance of a second treatment team at an MMTP may be an important factor in determining how closely the patient can be monitored. When two treatment teams are involved, each team should document a separate patient agreement not to abuse narcotics while receiving methadone, ${ }^{4}$ which could afford additional legal protection. Nurses and social workers from both teams should closely follow patients who are modifying their MMTP treatment of narcotics addiction to relieve chronic pain as well, in order to detect and address barriers to adherence and to overtreatment, especially new or worsening side effects that indicate risks for arrhythmia and sudden death (eg, see Shaiova et $\mathrm{al}^{22}$ ). In addition to biological or medical contraindications, MMTP treatment for pain management may be compromised by psychosocial barriers for medication adherence in specific populations, as documented in HIV-positive individuals with one or more psychiatric diagnoses. ${ }^{30}$

\section{Pharmacy clinics and retail pharmacies}

The criminal justice system and the neighborhood and regional environment, it may be argued, discriminate against many patients of lower socioeconomic status by denying them adequate access to pain and symptom relief. In particular, many physicians do not prescribe opioids, or in sufficient quantities, when there is any possibility of narcotics abuse or diversion because they fear being prosecuted by the DEA. ${ }^{3,4}$ Furthermore, inner-city pharmacies typically do not stock opioid medications due to the likelihood of assault, theft, and vandalism, ${ }^{5,6}$ and pharmacies in rural areas are rare. Given this austere reality, it is quite understandable that palliative care clinicians are often wary about innovations that seek to link, and limit, palliative care to contexts in which health providers are involved directly in efforts to control narcotics abuse and diversion.

However, in place of directly linking program components that discourage prescription drug abuse to outpatient palliative care (or to primary care services), health providers may express support for incorporating these types of innovative program linkages into the operations of retail pharmacies and pharmacy clinics. Professional self-interest may be an important factor. By associating with these innovative programs, health providers may feel more secure in the event of a DEA, police, or malpractice investigation. Pharmacists, in turn, may be receptive to adopt these program changes since they may become associated with perceptions of improved safety, which is important for attracting and retaining good pharmacy customers. Furthermore, pharmacists are increasingly informants about patients and their prescription drug use activity in state prescription drug monitoring program registries that operate in 38 states. ${ }^{31}$ (These state registries are tracked by local, state, and federal law enforcement agencies; some physicians also monitor their state registry to assess whether their patients are also seeking pain medications from other physicians or locations in the state in order to detect situations of potential prescription drug abuse or diversion. ${ }^{31}$ Pharmacists, however, can alert all physicians when their referrals appear on the registry.) Closely tied to pharmacist 
participation in state prescription monitoring programs, a pharmacist-directed program also provides a valuable opportunity for them to offer public health education to prevent prescription drug abuse. For instance, pharmacists can instruct patients and caregivers not to keep highly addictive and potentially lethal medications in the bathroom medicine cabinet or other open areas where they can be ingested or stolen by children, family members, or visitors.

How might innovative programs in pharmacy clinics and retail pharmacies actually be linked to the more limited efforts that we may reasonably expect of health providers from outpatient palliative care or primary care services in order to prevent and discourage prescription drug abuse? Community health providers who do not provide their own follow-up appointments to monitor patient medications, could be asked to do so or to require - as a condition of receiving a prescription for a controlled substance medication and any future refills - that their patients (or primary caregivers) attend periodic appointments at a pharmacy clinic, retail pharmacy, or other supportive community agency in order to provide pill counts (typically based on a labor-saving pillcounting machine). (These pharmacists and other providers would be notified in advance of any newly referred patients.) This condition for receiving a prescription from a community health provider would apply even to patients receiving medications delivered to their homes from online pharmacies. Bulk purchasing of online medications through a community health consortium, as discussed earlier, could subsidize costs to the pharmacy clinic or retail pharmacy, and patients purchasing directly from online pharmacies could still be charged a fee to cover the costs for pill counts and patient monitoring at the pharmacy clinic or retail pharmacy.

When pill counts are acceptable, appointments for pill counting could occur less frequently to minimize inconvenience, and a larger number of pills could be dispensed in prescription renewals.

If a pill count reveals too many missing pills, the health provider would be notified. Should the physician still decide to renew the prescription, the pharmacist would dispense a smaller quantity of pills and require more frequent pharmacy appointments to conduct pill counts and perhaps even to produce onsite a urine sample for lab analysis (described in the next paragraph). In any event, the pharmacist would notify the health provider to schedule a follow-up patient appointment in order to take a urine sample for lab analysis. (The health provider should not announce to the patient or caregiver in advance the plan to draw a urine sample in order to increase the chances for detecting drug abuse or misuse based on excessive or insufficient concentrations of the metabolized medication in the urine.) When there is evidence of potential abuse or diversion, the pharmacist would refuse to fill future prescriptions, and inform local police. In other situations, patients or caregivers who are actively abusing or diverting the medication may seek to bypass this local monitoring by purchasing controlled substances from online "rogue pharmacies," which do not even require a prescription. ${ }^{32}$ However, their failure to attend periodic pharmacy appointments would serve as a clue that patients, caregivers, family members, or friends could be abusing or diverting medication, and the pharmacist would notify the physician and perhaps the police.

When patients, their caregivers, or family members have past, current, or suspected involvement in drug abuse or diversion, both the health provider and pharmacy clinic should require that as part of the agreed plan for pain control, the patient will provide periodic urine samples onsite and under observation (ie, to prevent fraudulent urine samples; patients could be charged a fee). A safe medication dispensing center at a retail pharmacy could even perform this pharmacy clinic function by requiring a urine sample whenever these patients or their caregivers seek to fill a controlled substance medication prescription. Ideally, a single nursing assistant or aide would oversee the patient as they produce the urine sample in a private area; however, monitoring could also be carried out by the pharmacist or trained volunteer of the same gender as the patient. Either the pharmacist or the police officer providing clinic protection would deliver urine samples to the hospital for analysis, and the results would be reported to the health provider and pharmacist. Insufficient or excessive concentrations of the metabolized drug in the urine would provide a signal of drug abuse, misuse, or diversion by the patient, caregiver, family members, or social contacts, which may include supplementing pills through "doctor shopping" or using "rogue" mail-order pharmacies to obtain controlled substance medications without a prescription.

\section{Community agencies and social networks}

It is possible, however, that the use of assigned or off-duty police at retail pharmacies or pharmacy clinics may discourage some patients with active or past involvement in drug abuse or diversion, or with criminal records, who have a legitimate need for medications to relieve pain or other symptoms. On the other hand, it is common, and disturbing, that patients who abuse or divert drugs commonly manipulate physicians into prescribing higher doses or stronger types of opioids than are needed to achieve pain relief and control. ${ }^{3}$ Thus, there is a critical need to advocate for the extension of existing 
community residential drug treatment programs, MMTP clinics, medical outpatient clinics, visiting nurse services, and social work consultation - in order to conduct mandatory pill counting (which may involve use of a pill-counting machine), urine analyses, and psychosocial monitoring during home and/or agency visits - when patients receiving controlled substance medications have a personal or family history of drug abuse or diversion activities. These programs/settings should be included as part of the patient contract with their physician. Facilitating timely communication between the palliative care team and the interdisciplinary teams from these settings should become a priority.

Moreover, the scope for program linkage with existing community services may be wider than anticipated since there is precedence for nonmedical providers to engage in pill counting and psychosocial monitoring. Social service providers, for instance, already 1) oversee individuals with co-occurring mental health/mental retardation conditions who live together in a community residence, as they take medications for psychiatric and medical conditions (usually twice a day); and 2) conduct pill counts for these individuals on a daily basis.

Patients with reduced access to care are also more likely to delay treatment for pain. Respected and well established social networks in some communities, such as religious networks of clergy and church members, may be trusted by community residents when they need help accessing medical care. ${ }^{16}$ By conducting outreach and planning with these networks, palliative clinicians may anticipate "snowball" effects of new referrals of residents. Social workers, clergy, church members, and neighborhood leaders might consider how those who are uninsured, underinsured, or indigent, may be referred earlier in the disease course, when there may be more options for achieving and maintaining control over pain and symptoms. ${ }^{16}$

\section{Conclusions}

Societal expectations are likely to evolve for clinicians from retail and hospital pharmacies, pharmacy clinics, interdisciplinary health care teams, community drug-treatment programs, and social service agencies to assess and minimize the risks in meeting the twin goals of ensuring safe and affordable access to medications for palliative care while preventing prescription drug abuse and diversion. This article has presented a vision of geographic and service delivery innovations that begin to meet these competing bioethical goals. It is important to recognize that safe medication dispensing centers located within retail pharmacies or pharmacy clinics would operate as the centralized program component for hosting or coordinating most of the other interventions.
Pharmacists at safe medication dispensing centers would work with assigned or off-duty police officer guards at these centers to plan and oversee the safe storage of controlled substance medications in off-site community safe-deposit boxes. These pharmacists would also work with other professionals in the community - such as medical providers, social workers, hospital administrators and reimbursement officers, and drug abuse counselors - to: strengthen communication and cooperation with the prescribing medical provider; assist the prescribing medical provider in patient monitoring (ie, review the state prescription registry for evidence of doctor or pharmacy shopping; provide pill counts and urine samples); expand access to lower-cost formulations, and in some cases, TARD formulations of controlled substance medications; improve transportation access for underserved patients and caregivers to obtain prescriptions; and integrate community agencies and social networks as resources for patient support and monitoring. A couple of community program components may be hosted outside safe medication dispensing centers - developing medication purchasing cooperatives and expanding the role of inner-city MMTPs in palliative care. Increasingly, pharmacists, police, physicians, other medical providers, social workers, other mental health/drug abuse clinicians, and hospital administrators should work together as community leaders in this new area.

\section{Acknowledgments}

Portions of this work were supported by the National Institute of Mental Health (grant numbers 1R03MH064627-01, 5R03MH064627-02) and the Hartford Geriatric Social Work Faculty Scholar Initiative. The author is grateful to Dr Susan Murty, Associate Professor of Social Work at the University of Iowa for her receptive listening and enthusiastic support during the early development of several ideas in this commentary.

\section{Disclosure}

The author reports no conflicts of interest in this work.

\section{References}

1. King SA. Preventing prescription opioid abuse: new formulations - but who will benefit? Psychiatr Times [serial on the Internet]. 2009 Feb 1;26(2). http://www.psychiatrictimes.com/display/article/10168/ 1370716? verify $=0$. Accessed February 17, 2010.

2. Okie S. A flood of opioids, a rising tide of deaths. $N$ Engl J Med. 2010;363(21):1981-1985.

3. Selwyn P. Chapter 6: Pain management in substance abusers. In: Finkelstein R, Ramos SE, editors. Manual for Primary Care Providers: effectively caring for active substance users. New York: New York Academy of Medicine; 2002. Available from: http://www.nyhiv.com/ pdfs/NYAMmanual.pdf. Accessed February 17, 2011. 
4. Passik SD, Kirsh KL, McDonald MV, et al. A pilot survey of aberrant drug-taking attitudes and behaviors in samples of cancer and AIDS patients. J Pain Symptom Manage. 2000;19:274-286.

5. Morrison RS, Wallenstein S, Natale DK, Senzel RS, Huang L. "We don't carry that" - failure of pharmacies in predominantly nonwhite neighborhoods to stock opioid analgesics. $N$ Engl J Med. 2000;342(14):1023-1026.

6. Green C, Ndao-Brumblay S, West B, Washington T. Differences in prescription opioid analgesic availability: comparing minority and white pharmacies across Michigan. J Pain. 2005;6(10):689-699.

7. Tierney J. Trafficker or healer? And who's the victim? The New York Times. 2007 Mar 27. Available from: http://www.nytimes.com/2007/03/27/ science/27tier.html. Accessed February 17, 2011.

8. Rosenberg N, Goldstein J. Police suspect that drugs were goal of killer on L.I. The New York Times. 2011 Jun 21;Sect. A:22.

9. Hermitt A. Do I need a safe deposit box? What you should know about protecting your valuables [article on the Internet]. Available from: http://www.associatedcontent.com/article/990946/do_i_need_a_safe_ deposit_box.html?cat=3. Accessed July 7, 2011.

10. Bruce L. Anything goes in a safe-deposit box [article on the Internet] Available from: http://www.bankrate.com/brm/news/bank/20011023a. asp. Accessed July 5, 2011.

11. Crawford C. Bank safe deposit box rules and regulations [article on the Internet]. Available from: http://www.ehow.com/list_6857297_bankdeposit-box-rules-regulations.html. Accessed July 5, 2011.

12. SecureIDNews. Thinking outside the safe deposit box: Florida Credit Union members use biometric HandReaders [article on the Internet]. 2005 Mar 3. Available from: http://www.secureidnews. com/2005/03/03/thinking-outside-the-safe-deposit-box-florida-creditunion-members-use-biometric-handreaders/. Accessed July 5, 2011.

13. Lawrence J. Does mail-order pharmacy really deliver the goods? Managed Care [serial on the Internet]. 1998 Jun. http://www. managedcaremag.com/archives/9806/9806.backlash.shtml. Accessed February 17, 2011.

14. Francoeur RB, Murty S, Sandowski B. Chapter 13: Special considerations in rural and inner city areas. In: Altilio T, Otis-Green, S, editors. Oxford Textbook of Palliative Social Work. New York: Oxford University; 2011:125-140.

15. Morita T, Akechi T, Ikenaga M, et al. Late referrals to specialized palliative care service in Japan. J Clin Oncol. 2005;23:2637-2644.

16. Francoeur RB, Payne R, Raveis VH, Shim H. Palliative care in the inner-city: patient religious affiliation, underinsurance, and symptom attitude. Cancer. 2007;109(2 Suppl):425-434.

17. Johnson C. DEA crackdown hurts nursing home residents who need pain drugs. The Washington Post. 2009 Oct 29. Available from: http://www.washingtonpost.com/wp-dyn/content/article/2009/10/28/ AR2009102803146_pf.html. Accessed May 10, 2010.
18. Listening session before the Special Committee on Aging, United States Senate, One Hundred Eleventh Congress, Second Session. The War on Drugs Meets the War on Pain: nursing home patients caught in the crossfire. Washington, DC: US Government Printing Office; March 24, 2010; serial no. 111-16. Available from: http://www.gpoaccess.gov/ congress/index.html. Accessed October 22, 2010.

19. von Gunten CF, Bruera E, Pirrello RD, Portenoy RK. New opioids: expensive distractions or important additions to practice? J Palliat Med. 2010;13(5):505-511.

20. Bell J, Shearer J, Ryan A, et al. The acceptability, safety, and tolerability of methadone/naloxone in a 50:1 ratio. Exp Clin Psychopharmacol. 2009;17(3):146-153.

21. Ling W, Casadonte P, Bigelow G, et al. Buprenorphine implants for treatment of opioid dependence: a randomized controlled trial. JAMA. 2010;304(14):1576-1583.

22. Shaiova L, Berger A, Blinderman CD, et al. Consensus guideline on parenteral methadone use in pain and palliative care. Palliat Support Care. 2008;6:165-176.

23. Arnaert A, Ciccotosto G. Response phases in methadone treatment for chronic nonmalignant pain. Pain Manage Nurs. 2006;7(1):23-30.

24. Blinderman CD, Sekine R, Zhang B, Nillson M, Shaiova L. Methadone as an analgesic for patients with chronic pain in methadone maintenance treatment programs. J Opioid Manag. 2009;5(2):107-114.

25. Joranson DE. Is methadone maintenance the last resort for some chronic pain patients? APS Bulletin. 1997;7(5):1, 4-5.

26. Rolfs RT, Johnson E, Williams NJ, Sundwall DN. Utah clinical guidelines on prescribing opioids for treatment of pain. J Pain Palliat Care Pharmacother. 2010;24:219-235.

27. Chou R, Fanciullo G, Fine PG, Miaskowski C, Passik S, Portenoy P. Opioids for chronic noncancer pain: prediction and identification of aberrant drug-related behaviors: a review of the evidence for an American Pain Society and American Academy of Pain Medicine clinical practice guideline. J Pain. 2009;10:131-146.

28. Newman R. Prescription opioids and overdose deaths. JAMA. 2009; 301(17): 1767.

29. Nicholson AB. Methadone for cancer pain. Cochrane Database Syst Rev. 2007;4:CD003971.

30. Palmer NB, Salcedo J, Miller AL, Winiarski M, Arno P. Psychiatric and social barriers to HIV medication adherence in a triply diagnosed methadone population. AIDS Patient Care STDs. 2003;17(12): 635-644.

31. Beck M. Doctors' challenge: how real is that pain? The Wall Street Journal. $2011 \mathrm{Jul} 5$. Available from: http://online.wsj.com/article/SB1 0001424052702304450604576419810283786774.html. Accessed July $5,2011$.

32. PharmacyChecker.com. Find the best drug prices from verified online pharmacies [homepage on the Internet]. Available from: http://www. pharmacychecker.com. Accessed July 5, 2011.
Risk Management and Healthcare Policy

\section{Publish your work in this journal}

Risk Management and Healthcare Policy is an international, peerreviewed, open access journal focusing on all aspects of public health, policy, and preventative measures to promote good health and improve morbidity and mortality in the population. The journal welcomes submitted papers covering original research, basic science, clinical \& epidemio-

\section{Dovepress}

logical studies, reviews and evaluations, guidelines, expert opinion and commentary, case reports and extended reports. The manuscript management system is completely online and includes a very quick and fair peerreview system, which is all easy to use. Visit http://www.dovepress.com/ testimonials.php to read real quotes from published authors. 